\title{
DISCLAIMER
}

This report was prepared as an account of work sponsored by an agency of the United States Government. Neither the United States Government nor any agency thereof, nor any of their employees, makes any warranty, express or implied, or assumes any legal liability or responsibility for the accuracy, completeness, or usefulness of any information, apparatus, product, or process disclosed, or represents that its use would not infringe privately owned rights. Reference herein to any specific commercial product, process, or service by trade name, trademark, manufacturer, or otherwise does not necessarily constitute or imply its endorsement, recommendation, or favoring by the United States Government or any agency thereof. The views and opinions of authors expressed herein do not necessarily state or reflect those of the United States Government or any agency thereof.

RAL-94-119

GPP-94-34

)OE/ER/40757-057

November 1994

\section{Signatures of Higgs-Triplet Representations at $\mathrm{TeV}-e^{+} e^{-}$Colliders}

Kingman Cheung ${ }^{a}$, Roger J.N. Phillips ${ }^{b}$, and Apostolos Pilaftsis ${ }^{b}$

a University of Texas at Austin, Center for Particle Physics, Austin, TX 78712, USA

${ }^{b}$ Rutherford Appleton Laboratory, Chilton, Didcot, Oxon, OX11 OQX, UK

\begin{abstract}
We investigate the potential of future $\mathrm{TeV}$ linear $e^{+} e^{-}$colliders to observe singlycharged Higgs bosons $\left(H^{ \pm}\right)$via the coupling $H^{ \pm} W^{\mp} Z$, which would signal the existence of exotic Higgs representations. In the context of a Higgs-triplet model compatible with the electroweak oblique parameters, we estimate the cross section for producing charged Higgstriplet bosons that couple predominantly to $W$ and $Z$ bosons in $0.5-2 \mathrm{TeV}-e^{+} e^{-}$colliders. The principal backgrounds are evaluated and the viability of the signal is discussed and illustrated.
\end{abstract}


The physics potential of a $\mathrm{TeV}-e^{+} e^{-}$collider, such as the proposed next linear collider (NLC) planned to operate with a centre-of-mass energy of $500 \mathrm{GeV}[1]$, includes the exploration of the parameter space of theories beyond the minimal Standard Model (SM). Theories beyond the SM usually predict the existence of charged Higgs bosons $\left(H^{ \pm}\right)$[2]. In particular, if charged Higgs bosons are produced via a sizeable $H^{ \pm} W^{\mp} Z$ coupling, this alone can reveal the origin of the charged Higgs boson, i.e., as a member of a Higgs-triplet realization beyond the SM. In extensions of the SM with Higgs doublets and singlets, the coupling $H^{ \pm} W^{\mp} Z$ vanishes at tree level and can only be generated at one-loop level [3, 4]. The reason for the vanishing of the $H^{ \pm} W^{\mp} Z$ coupling in the Lagrangian is rather technical and depends upon the hypercharge $(Y)$ and weak-isospin assignments of the Higgs representations introduced in the model. In fact, the $W^{ \pm}$and $Z$ bosons couple through the covariant derivative to the charged Higgs and would-be Goldstone $\left(G^{ \pm}\right)$bosons, and a tree-level coupling to the singlet Higgs fields $(Y=0)$ is therefore prohibited. On the other hand, in models with complex Higgs doublets $\Phi_{i}(Y=1)$, the vertex $H^{ \pm} W^{\mp} Z$ is proportional to $T_{-}\left\langle\Phi_{i}\right\rangle$ and hence vanishes $\left[T_{ \pm}=\frac{1}{2}\left(\sigma_{1} \pm \sigma_{2}\right)\right.$, with the Pauli matrices denoted by $\left.\sigma_{1,2,3}\right]$, whereas $G^{ \pm} W^{\mp} Z \propto T_{+}\left\langle\Phi_{i}\right\rangle$ is non-zero as should be the case in a renormalizable extension of the SM. In multi-Higgs doublet models, the resulting strength of the loopinduced $H^{+} W^{-} Z$-coupling turns out to be rather small of the order of $10^{-2}$ relative to the SM vertex $H W^{+} W^{-}$. A large $H^{ \pm} W^{\mp} Z$ coupling is therefore an indicator of exotic triplet or higher Higgs representations beyond the SM; searches for experimental signatures of this coupling will offer unique tests for the presence of such exotic representations.

In the context of theories containing $Y=2$ Higgs-triplet fields, our aim is to show that $\mathrm{TeV}-e^{+} e^{-}$colliders are capable of differentiating whether the charged Higgs bosons belong to a triplet or doublet representation after taking into account the SM background. Such a distinction is harder to achieve at hadron colliders; searches there for doublet charged Higgs bosons have been discussed [5]. Complex triplet representations also predict doubly charged Higgs bosons (i.e. $\mathrm{H}^{++}$); we shall not address their signatures here, but refer the reader to Ref. [6] for $\mathrm{H}^{++}$signals at hadron colliders and Ref. [7] for $\mathrm{H}^{--}$production at $e^{-} e^{-}$linear colliders.

In models with Higgs triplets, one has to face difficulties coming from large contributions to the electroweak parameters $S, T$, and $U$ [8] (generalized to $V, W$, and $X$ [9]). 


\section{DISCLAIMER}

Portions of this document may be illegible in electronic image products. Images are produced from the best available original document. 
Especially, compatibility with the Veltman parameter $\rho(\alpha T)[10]$ and the absence of large flavour-changing neutral currents suggest that the neutral component of the left-handed triplet should possess an unnaturally small vacuum expectation value of the order of $\mathrm{eV}$. An interesting scenario that avoids this problem was considered by Galison [11], and Georgi and Machacek [12]. They introduced more than one triplet field into the model and imposed an $S U(2)$ custodial symmetry on the vacuum expectation values and hypercharges of the Higgs multiplets to ensure $\rho=1$ at tree level. This scenario was further analyzed by Chanowitz and Golden [13], who examined stability conditions of the $S U(2)$ custodial symmetry in the Higgs potential under higher order quantum corrections. To be more precise, the model under discussion consists of the usual SM $Y=1$ complex doublet $\Phi$, plus one real $Y=0$ and one complex $Y=2$ triplet given by

$$
\Delta=\left(\begin{array}{ccc}
\delta^{0} & \chi^{+} & \delta^{++} \\
\delta^{-} & \chi^{0} & \delta^{+} \\
\delta^{--} & \chi^{-} & \delta^{0 *}
\end{array}\right) .
$$

Among the various Higgs fields that the model predicts, there exist charged Higgs-triplet bosons $H^{ \pm}$(sometimes denoted as $H_{5}^{ \pm}$), which have no-tree level couplings to fermions. In addition to $H^{ \pm}$, the model also contains charged Higgs-doublet bosons $H^{\prime \pm}$ (also called $\left.H_{3}^{ \pm}\right)$that do not couple to gauge bosons in the Born approximation. Specifically, after diagonalizing the charged Higgs-boson matrix by assuming that the $S U(2)$ custodial symmetry is preserved, they are identified as

$$
H^{ \pm}=\sqrt{\frac{1}{2}}\left(\delta^{ \pm}-\chi^{ \pm}\right), \quad H^{ \pm}=c_{H} \sqrt{\frac{1}{2}}\left(\delta^{ \pm}+\chi^{ \pm}\right)-s_{H} \phi^{ \pm},
$$

where $\phi^{+}$is the charged-field component of the Higgs doublet $\Phi$ and $s_{H}=\sqrt{1-c_{H}^{2}}$ is the sine of a doublet-triplet mixing angle defined as

$$
s_{H}=\sqrt{\frac{8 v_{T}^{2}}{v_{D}^{2}+8 v_{T}^{2}}}
$$

with $v_{D} / \sqrt{2}=\left\langle\phi^{0}\right\rangle$ and $v_{T}=\left\langle\delta^{0}\right\rangle=\left\langle\chi^{0}\right\rangle$. The SM vacuum expectation value is then related to $v_{D}$ and $v_{T}$ via $v^{2}=v_{D}^{2}+8 v_{T}^{2}$. The corresponding vertex $H^{+} W^{-} Z$ is then given by [14]

$$
\mathcal{L}_{\text {int }}=-g_{w} \frac{s_{H}}{c_{w}} M_{W} H^{+} W^{-\mu} Z_{\mu}+\text { H.c. }
$$


where $g_{w}$ is the usual $S U(2)_{L}$ electroweak coupling constant, $c_{w}^{2}=1-s_{w}^{2}=M_{W}^{2} / M_{Z}^{2}$ and $s_{w}^{2}=\sin ^{2} \theta_{w}$ is the sine-square of the Weinberg angle. Due to electromagnetic gauge invariance, the coupling $H^{ \pm} W^{\mp} \gamma$ is absent at tree level. As emphasized earlier, we are interested in a large $H^{ \pm} W^{\mp} Z$ coupling that will unavoidably signify the triplet nature of the charged Higgs bosons $H^{ \pm}$. This can only be the case if $s_{H} \sim 1$ or equivalently $v_{T} \sim v_{D}$, which is considered to be a natural scenario. In the limit of our interest $\left(s_{H} \rightarrow 1\right)$, the only interactions of $H^{ \pm}$with other fields that survive in the Lagrangian are those between the so-called fiveplet members $\left(H_{5}^{--}, H^{-}, H_{5}^{0}, H^{+}, H_{5}^{++}\right.$in the notation of [15]), i.e., the couplings $H_{5}^{0} H^{-} W^{+}, H^{+} H_{5}^{--} W^{+}, H^{+} H^{-} Z, H^{-} H^{+} \gamma$, and the one given by Eq. (4). There is also a tri-Higgs vertex $H^{+} H^{\prime} H_{3}^{0}$, which depends crucially on the details of the Higgs potential. Thus, for some specific choice of parameters, $H_{3}^{0}$ can be heavier than $H^{+}$and $H^{+} \nrightarrow H^{\prime+} H_{3}^{0}$. An exhaustive list of the Feynman rules containing all the Higgs particles involved in this model can be found in Ref. [15]. Furthermore, as an effect of the $S U(2)$ custodial symmetry, all fiveplet members are degenerate in mass and so the only dominant decay mode is $H^{+} \rightarrow W^{+} Z$. The partial width of this decay channel is given by

$$
\begin{aligned}
\Gamma\left(H^{+} \rightarrow W^{+} Z\right)= & \frac{\alpha_{w}}{16}\left(\frac{s_{H}^{2}}{1}\right) M_{H} \lambda^{1 / 2}\left(M_{H}^{2} / M_{W}^{2}, 1 / c_{w}^{2}, 1\right)\left[1+x_{W}^{2}+x_{Z}^{2}\right. \\
& \left.-2 x_{W}-2 x_{Z}+10 x_{W} x_{Z}\right],
\end{aligned}
$$

with $\alpha_{w}=g_{w}^{2} / 4 \pi, \lambda(x, y, z)=(x-y-z)^{2}-4 y z, x_{W}=M_{W}^{2} / M_{H}^{2}$, and $x_{Z}=M_{Z}^{2} / M_{H}^{2}$. Of course, if such a scenario were embedded in a grand unified theory (GUT), one would have to cope with the known gauge-hierarchy problem or problems related to the existence of a unification point at the GUT scale $M_{X}$. Solutions to these problems may be achieved by considering a supersymmetric GUT version that contains our low-energy model [15]. One may therefore expect that additional supersymmetric scalars will be present in the theory and give rise to new decay modes for a very heavy charged Higgs boson with mass of order $1 \mathrm{TeV}$. For our present illustrations, however, it is reasonable to consider a scenario in which $B\left(H^{+} \rightarrow W^{+} Z\right) \simeq 1$ for charged Higgs masses $M_{H} \lesssim 600 \mathrm{GeV}$ and $s_{H} \simeq 1$.

There are two preferred channels for hunting the charged-triplet Higgs at $\mathrm{TeV}-e^{+} e^{-}$ colliders: (i) $e^{+} e^{-} \rightarrow Z^{*} \rightarrow W^{-} H^{+}$and (ii) $e^{+} e^{-} \rightarrow W^{+*} Z^{*} e^{-} \bar{\nu}_{e} \rightarrow H^{+} e^{-} \bar{\nu}_{e}$ (illustrated in Fig. 1), both of which depend on the $W^{ \pm} Z H^{\mp}$ vertex. 
(i) The Bjorken-type process $e^{+} e^{-} \rightarrow Z^{*} \rightarrow W^{-} H^{+}$

The Feynman diagram is depicted in Fig. 1(i). The total cross section for $e^{+} e^{-} \rightarrow Z^{*} \rightarrow$ $W^{ \pm *} H^{\mp} \rightarrow f \bar{f}^{\prime} H^{\mp}$, where $W^{ \pm *}$ denotes an off-shell $W$ boson and $f \bar{f}^{\prime}$ is any fermion pair from the $W$ decay, is given by

$$
\sigma_{t o t}(s)=\frac{\alpha_{w}^{3} N_{c} s_{H}^{2}}{192 c_{w}^{4} s} \frac{\left(1+v_{e}^{2}\right) M_{W}^{2}}{\left(s-M_{Z}^{2}\right)^{2}+M_{Z}^{2} \Gamma_{Z}^{2}} I\left(s, M_{H}^{2}\right),
$$

where $v_{e}=1-4 s_{w}^{2}$, the colour factor $N_{C}=3$ (1) for quarks (leptons), and

$$
\begin{aligned}
I\left(s, M_{H}^{2}\right)= & \operatorname{Re} J\left(2 M_{H} \sqrt{s}, s+M_{H}^{2}-M_{W}^{2}-i \Gamma_{W} M_{W}, s+M_{H}^{2}\right) \\
& +\frac{M_{W}}{\Gamma_{W}} \operatorname{Im} J\left(2 M_{H} \sqrt{s}, s+M_{H}^{2}-M_{W}^{2}-i \Gamma_{W} M_{W}, s+M_{H}^{2}\right) .
\end{aligned}
$$

The function $J(\alpha, \beta, \gamma)$ is defined as

$$
\begin{aligned}
J(\alpha, \beta, \gamma)= & \int_{\gamma}^{\alpha} d x \frac{\sqrt{x^{2}-\alpha^{2}}}{x-\beta}=-\sqrt{\gamma^{2}-\alpha^{2}}-i \pi \sqrt{\beta^{2}-\alpha^{2}}+\left(\beta+\sqrt{\beta^{2}-\alpha^{2}}\right) \ln \alpha \\
& -\beta \ln \left(\gamma+\sqrt{\gamma^{2}-\alpha^{2}}\right)-\sqrt{\beta^{2}-\alpha^{2}} \ln \left(\frac{\beta \gamma-\alpha^{2}-\sqrt{\beta^{2}-\alpha^{2}} \sqrt{\gamma^{2}-\alpha^{2}}}{\gamma-\beta}\right) .
\end{aligned}
$$

In case of complex arguments, the function $J(\alpha, \beta, \gamma)$ should be continued analytically. In Fig. 2, we have plotted the total cross section (summing $H^{+}$and $H^{-}$channels) as a function of the charged Higgs mass at center-of-mass energies $\sqrt{s}=0.5,1,1.5$ and $2 \mathrm{TeV}$. We also summed over all the fermion pairs $f \bar{f}^{\prime}$. Since $H^{ \pm}$decays into $W$ and $Z$ bosons, the process of interest is $e^{+} e^{-} \rightarrow f \bar{f}^{\prime} W^{ \pm} Z$, in which the vector bosons may be identified via their leptonic decays into electrons and muons. Obviously, the irreducible background is the SM production of $e^{+} e^{-} \rightarrow W^{+} W^{-} Z$. The leptonic branching fractions are:

$$
B\left(W^{-} \rightarrow e^{-}, \mu^{-}+X_{\nu}\right) \simeq 0.26, \quad B\left(Z \rightarrow e^{-} e^{+}, \mu^{-} \mu^{+}\right) \simeq 0.067
$$

where we have included the modes $W^{-} \rightarrow \tau^{-} \bar{\nu}_{\tau} \rightarrow e^{-} / \mu^{-} \bar{\nu}_{e / \mu} \nu_{\tau} \bar{\nu}_{\tau}$, so the quantity $X_{\nu}$ denotes either one or three neutrinos. From these branching fractions and the cross sections of Fig. 2, we see that pure leptonic signals from charged Higgs production via this process are very small, and decrease as c.m.s. energy $\sqrt{s}$ increases as indicated by the $1 / s$ factor in Eq. (6). We shall therefore henceforth focus our attention mainly on the more promising $W Z$ fusion process, but we will take account of small contributions from the Bjorken process at $\sqrt{s}=0.5 \mathrm{TeV}$, where they are not negligible. 
(ii) The fusion process $e^{+} e^{-} \rightarrow W^{+*} Z^{*} e^{-} \bar{\nu}_{e} \rightarrow H^{+} e^{-} \bar{\nu}_{e}$

This reaction depicted in Fig. 1(ii) offers larger signals than the previous process. The total cross section can be written

$$
\sigma_{t o t}(s)=\frac{1}{1024 \pi^{4} s^{2}} \int \frac{d s_{2} d t_{1} d t_{2} d s_{1}}{\sqrt{-\Delta_{4}\left(s, s_{1}, s_{2}, t_{1}, t_{2}\right)}} \overline{|\langle\mathcal{T}\rangle|^{2}},
$$

where the squared transition element averaged over the spins of the initial states is

$$
\overline{|\mathcal{T}\rangle \mid}^{2}=\frac{g_{w}^{2} M_{W}^{2} s_{H}^{2} g_{L}^{(w) 2}}{c_{w}^{2}\left(t_{1}-M_{Z}^{2}\right)^{2}\left(t_{2}-M_{W}^{2}\right)^{2}}\left[g_{L}^{(z) 2} s\left(s-s_{1}-s_{2}+M_{H}^{2}\right)+g_{R}^{(z) 2}\left(s-s_{1}+t_{2}\right)\left(s-s_{2}+t_{1}\right)\right] \text {, }
$$

and the coupling constants $g_{L}^{(w)}, g_{L, R}^{(z)}$ are given by

$$
\begin{aligned}
& g_{L, R}^{(i)}=g_{V}^{(i)} \mp g_{A}^{(i)}, \quad i=w, z \\
& g_{V}^{(w)}=-g_{A}^{(w)}=\frac{g_{w}}{2 \sqrt{2}}, \quad g_{V}^{(z)}=-\frac{g_{w}}{4 c_{w}}\left(1-4 s_{w}^{2}\right), \quad g_{A}^{(z)}=\frac{g_{w}}{4 c_{w}} .
\end{aligned}
$$

Here, the superscripts $(w)$ and $(z)$ refer to the production vertices of a virtual $W$ and $Z$ boson, respectively. Furthermore, $s, s_{1}, s_{2}, t_{1}$, and $t_{2}$ in Eq. (11) are the usual Mandelstam variables defined as follows:

$$
\begin{aligned}
& s=\left(k_{e^{-}}+k_{e^{+}}\right)^{2}, \quad t_{1}=\left(p_{e^{-}}-k_{e^{-}}\right)^{2}, \quad t_{2}=\left(p_{\nu}-k_{e^{+}}\right)^{2}, \\
& s_{1}=\left(p_{e^{-}}+p_{H}\right)^{2}, \quad s_{2}=\left(p_{\nu}+p_{H}\right)^{2} \text {. }
\end{aligned}
$$

The phase-space limits of the Mandelstam variables listed in Eq. (13) as well as the definition of the kinematic function $\Delta_{4}$ in Eq. (10) can be found in Ref. [16]. In Fig. 3, we show the computed total cross section as a function of the charged Higgs boson mass $M_{H}$ at $\sqrt{s}=0.5,1,1.5$, and $2 \mathrm{TeV}$. Unlike the Bjorken-type process this channel has a cross section increasing with $\sqrt{s}$.

The signal of interest is $e^{+} e^{-} \rightarrow e^{\mp} \nu H^{ \pm} \rightarrow e^{\mp} \nu W^{ \pm} Z$ with leptonic decays; we therefore concentrate on the channel

$$
e^{+} e^{-} \rightarrow e \nu W Z \rightarrow e \nu(\ell \nu)\left(\ell^{\prime} \bar{\ell}^{\prime}\right)
$$

where $\ell, \ell^{\prime}$ denote $e$ or $\mu$. It is understood that decays $W \rightarrow \tau \nu \rightarrow \ell \nu \nu \nu$ are always included, since they are practically impossible to be distinguished experimentally from the 
direct leptons in $W \rightarrow \ell \nu$, but decays $Z \rightarrow \tau \tau \rightarrow \ell \ell \nu \nu \nu \nu$ can be excluded because the dilepton invariant mass is generally much less than $M_{Z}$. The $W Z$-fusion process then has net branching fraction 0.017 , that multiplies the cross section of Fig. 3 to give the cross section in this leptonic channel.

The main characteristics of the $W Z$-fusion four-lepton signal are: three hard central leptons from $W$ and $Z$ decay; one scattered beam $e^{ \pm}$; two of the leptons reconstruct the $Z$ boson; and the two undetectable neutrinos give large missing $p_{T}$. Note that the Bjorken process also contributes in this channel, albeit at a low level, and must be added to the final signal.

We must now discuss the main SM backgrounds in the above channel, together with possible kinematic cuts to reduce them with minimal loss of signal. These backgrounds are:

(a) $e^{+} e^{-} \rightarrow W^{+} W^{-} Z$, with one $W$ boson decaying to $e$ or $\mu$ and the other only to $e$. This background cannot easily be removed and must be calculated in detail, though the cross section decreases as $\sqrt{s}$ increases. It is part of the annihilation channel $e^{+} e^{-} \rightarrow$ $W^{+} W^{-} Z^{*} \rightarrow W^{+} W^{-} \ell \bar{\ell}(\ell=e, \mu)$. Actually, it can also be viewed as part of $e^{+} e^{-} \rightarrow$ $W^{-} Z W^{+*} \rightarrow W^{-} Z \ell^{+} \nu$, or $e^{+} e^{-} \rightarrow W^{+} Z W^{-*} \rightarrow W^{+} Z \ell^{-} \bar{\nu}$. To avoid double counting we include it in the $e^{+} e^{-} \rightarrow W^{-} Z W^{+*} \rightarrow W^{-} Z \ell^{+} \nu$ calculation.

(b) $e^{+} e^{-} \rightarrow e^{+} e^{-} W^{+} W^{-}$with leptonic $W$ decays. This background refers only to the scattering channel contribution; the annihilation channel is already included in process (a). The total cross section of this production is very large, of order $2 \mathrm{pb}$ at $\sqrt{s}=1.5 \mathrm{TeV}$ due to the double photon-exchange diagrams. Fortunately, this huge cross section can be substantially reduced by requiring both the scattered beam electron and positron to be away from the beam direction (e.g. requiring $\left|\cos \theta_{e}\right|<0.98$ ), and by constraining the invariant mass of one lepton pair to be around the $Z$ mass while the invariant mass of the other pair is larger than $M_{Z}+10 \mathrm{GeV}$. After all these requirements this background remains non-negligible, so we include it in our analysis.

(c) $e^{+} e^{-} \rightarrow e^{ \pm} W^{\mp} Z \nu$, followed by the subsequent decays $W^{ \pm} \rightarrow e^{ \pm}, \mu^{ \pm} X_{\nu}$ and $Z \rightarrow e e, \mu \mu$. The Feynman graphs of this SM reaction may be found in Fig. 4 of Ref. [17]. This process refers only to the scattering channel, while the annihilation channel is already included in 
process (a). The total cross section of this process is also very large due to the photonexchange diagrams. The cross section can be reduced by excluding electrons close to the beam, but the reduction is less than in $e^{+} e^{-} \rightarrow e^{+} e^{-} W^{+} W^{-}$and it remains a major background to our signal. Since this process $[17,18]$ has very similar features to our signal a more sophisticated investigation of kinematic variables is needed. Thanks to the difference that there are no resonance graphs with a heavy charged Higgs boson in this background, we can exploit the invariant mass of the three charged leptons that decay from the $W Z$. While the background should be smooth in this distribution, the signal should be concentrated in a limited range depending on the charged Higgs-boson mass.

(d) $e^{+} e^{-} \rightarrow Z Z$ with leptonic $Z$ decays. The case where both $Z$ decay directly to $e$ or $\mu$ pairs can be suppressed by requiring that only one pair has invariant mass near $M_{Z}$, and by requiring a large missing transverse momentum $p_{T}$. There remains a contribution where the second $Z$ decays via $Z \rightarrow \tau^{+} \tau^{-} \rightarrow e e, e \mu+X_{\nu}$; this is important only at $\sqrt{s}=0.5$ $\mathrm{TeV}$, where $\sigma_{b} \simeq 0.2 \mathrm{fb}$, and can be removed completely by requiring that the second pair of leptons have invariant mass greater than $M_{Z}$.

(e) $e^{+} e^{-} \rightarrow Z e^{+} e^{-}$with $Z$ decaying directly to $e e$ or $\mu \mu$. This background refers to the scattering channel (process (d) already includes the major annihilation channel). The total production cross section is of order $1 \mathrm{pb}$ at $\sqrt{s}=0.5-2 \mathrm{TeV}$ [7], including the $Z$ decay branching ratio. It is reduced to the level of $1 \mathrm{fb}$ by cutting out leptons at small beam angles, and can be finally eliminated by a missing transverse momentum cut.

(f) $e^{+} e^{-} \rightarrow Z Z Z^{*}, Z Z \gamma_{\gamma^{*}}$. These annihilation processes are of higher order than process (d) and therefore generally smaller. If the final $Z$ bosons and/or the off-shell photon go to $\ell^{+} \ell^{-}$, $\nu \bar{\nu}$ and $e^{+} e^{-}$, respectively, they contribute to the same final states as $(\mathrm{g})$ and $(\mathrm{h})$ below.

(g) $e^{+} e^{-} \rightarrow Z Z e^{+} e^{-}$scattering, with one $Z$ decaying invisibly. This can fake signal events but is at least an order of magnitude smaller than (c) and the small-angle cut on both the scattered $e^{+}$and $e^{-}$reduce it to a negligible level. For example, at $1.5 \mathrm{TeV}$ this background is only of order $10^{-3} \mathrm{fb}$.

(h) $e^{+} e^{-} \rightarrow Z Z \nu \nu$ scattering, with one $Z$ boson decaying via $\tau$ leptons into $e^{+} e^{-} \nu \nu \nu \nu$ or $e \mu \nu \nu \nu \nu$. This background can be removed by requiring the invariant mass of $e^{+} e^{-} / e \mu$ to 
be larger than $M_{Z}$.

Thus the only major backgrounds are (a), (b), and (c).

Our strategies to select the signal and minimize these backgrounds are as follows.

- We select events with exactly four charged leptons in the final state (no hadrons), at least one of which must be $e^{ \pm}$, and impose the following lepton acceptance cuts:

$$
p_{T}^{\ell}>10 \mathrm{GeV} \quad \text { and } \quad\left|\cos \theta_{\ell}\right|<0.98
$$

where $\theta_{\ell}$ is the angle between the lepton and the beam direction.

- Since two of the four charged leptons should come from a $Z$ boson, we require one pair of oppositely charged leptons of the same flavour to reconstruct the $Z$ mass in the range

$$
M_{Z}-10 \mathrm{GeV}<M\left(\ell^{+} \ell^{-}\right)<M_{Z}+10 \mathrm{GeV}
$$

- For the other pair of leptons (which should come from $W$ decay and a scattered $\left.e^{-} / e^{+}\right)$, we require them to have opposite charges, one of them to be $e^{ \pm}$, and their invariant mass $M(e \ell)$ to be above the $Z$ range:

$$
M(e \ell)>M_{Z}+10 \mathrm{GeV} \text {. }
$$

- We impose a missing transverse momentum cut

$$
\not p_{T}>30 \mathrm{GeV} \text {. }
$$

- We attempt to form the invariant mass of the two leptons, which reconstruct the $Z$ boson, plus the lepton from the $W$ decay. For $e \mu Z$ final states it is uniquely determined that $M(\mu Z)$ is the correct combination. But for eeZ final states (half of our signal) the choice is ambiguous; here we choose the minimum of the two invariant masses $M(e Z)$, denoted by $M(\ell \ell \ell-\min )$. In the case of the signal, $M(\ell \ell \ell-\min )$ turns out to have a distribution very similar to the "correct" invariant mass $M(\mu Z)$ in the e $\mu Z$ channel; both have the same sharp upper limit

$$
M^{2}<\frac{1}{2}\left[M_{H}^{2}+M_{Z}^{2}-M_{W}^{2}+\lambda^{1 / 2}\left(M_{H}^{2}, M_{Z}^{2}, M_{W}^{2}\right)\right]<M_{H}^{2}
$$

The lower limit on $M(\mu Z)$ is found by reversing the sign of $\lambda^{1 / 2}$ above. This variable is intended to distinguish further between signal and background. 
A possible additional strategy would be to select only $e \mu Z$ in the final state. This

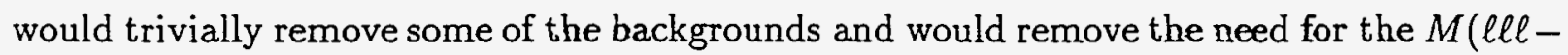
min) variable. However, the signal would then be halved and the major backgrounds would remain, reduced by no more than the same factor 2 . We do not choose this option here.

We have computed the triplet-Higgs signal and the main backgrounds with the above acceptance criteria, using Monte Carlo methods. The signal calculations are based on spinor trace techniques; the $H^{ \pm} \rightarrow W^{ \pm} Z \rightarrow \ell^{ \pm} \nu \ell^{\prime} \bar{\ell}^{\prime}$ decay trace is analogous to the production trace, with appropriate crossings; the effects of $W \rightarrow \tau \nu \rightarrow \ell \nu \nu \nu$ cascade decays are included by the methods of Ref.[19]. The background calculations are based on helicity amplitude techniques, extending the codes originally developed in Ref.[17]. We have restricted ourselves to masses $M_{H}>M_{W}+M_{Z}$, for which on-shell $H^{ \pm} \rightarrow W^{ \pm} Z$ decays are possible. Our integrated cross section results are exhibited in Table 1. Several comments should be made.

(i) The signals do not rise monotonically with energy, unlike the uncut cross sections in Fig. 3. This is mostly because of the angular cut on the scattered beam electron or positron, that removes a larger fraction of electrons at higher energy. The signal would increase if this cut were relaxed, and ideally one might consider different cuts for different energies; however, the background would increase even more (and there are also practical difficulties in detecting at small angles in linear colliders), so we have not pursued this option.

(ii) The other cuts do not cost more at higher energy. The cut on the two non- $Z$ lepton invariant mass is in fact the most costly at the lowest energy, $\sqrt{s}=0.5 \mathrm{TeV}$, where it typically halves the signal; this is understandable, because the scattered beam electron is less energetic at lower $s$.

(iii) The Bjorken process contributes significantly at the lowest energy only, giving $20 \%$ (60\%) of our signal for $M_{H}=175 \mathrm{GeV}(350 \mathrm{GeV})$ there.

(iv) $W \rightarrow \tau \nu \rightarrow \ell \nu \nu \nu$ decays give between $4 \%$ and $12 \%$ of our signal, losing a larger fraction to the cuts, especially at lower energies.

Are such signals detectable above the backgrounds? Assuming annual luminosity $50 \mathrm{fb}^{-1}$ at each energy, and a net lepton identification efficiency of $60 \%$ or more per event, we see the possibilities at $\sqrt{s}=0.5 \mathrm{TeV}$ are rather limited; however, charged Higgs-triplet bosons with masses up to about $400 \mathrm{GeV}$ might eventually be detectable for large values 
of the mixing angle $s_{H}$, at the higher energies. For example, at $\sqrt{s}=1.5 \mathrm{TeV}$ with $M_{H}=175-400 \mathrm{GeV}$ and $s_{H} \sim 1$, there would be about 8-12 signal events on top of 9 background events per year, giving a somewhat significant excess in one year.

If the presence of a signal can be detected as an excess of events over the expected background, its origin as a $W Z$ resonance can be confirmed and the Higgs mass extracted by a study of the trilepton invariant mass distributions. In Fig. 4, we illustrate the $M(\ell \ell \ell-$ min) distribution at $\sqrt{s}=1.5 \mathrm{GeV}$, for triplet Higgs masses 200,300, 400, $500 \mathrm{GeV}$; the case of $M(\mu Z)$, that can only be defined in $e \mu Z$ channels, is rather similar. We see that the signal and background have quite different distributions. In the case $M_{H}=200 \mathrm{GeV}$, the narrow signal peak between $M(\ell \ell \ell-\min )=100$ and $M(\ell \ell \ell-\min )=180 \mathrm{GeV}$ is particularly striking, compared to the broad background continuum. For higher Higgs masses, the signal peak is broader but nevertheless has a sharp upper limit and changes the net distribution shape in a very significant way. For estimating the significance of the signal, we should compare only with the background events directly under the signal peak; this improves the numerical significance of our signal. For example, for $M_{H}=200 \mathrm{GeV}$ at $\sqrt{s}=1.5 \mathrm{TeV}$ with $50 \mathrm{fb}^{-1}$ luminosity, we should compare 12 signal events in the Higgs peak with about 4 background events under this peak (see Fig. 4), rather than the total of 9 background events altogether.

We now briefly discuss the effects of initial state radiation (bremsstrahlung and beamstrahlung), that are not included in our analysis above. Both bremsstrahlung and beamstrahlung reduce the center-of-mass energy $\sqrt{s}$ to an effective center-of-mass energy $\sqrt{\hat{s}}$, while beamstrahlung at $e^{+} e^{-}$colliders also increases the effective luminosities. The effect of beamstrahlung on the effective luminosities at various $e^{+} e^{-}$collider designs can be found in Ref. [20]; the increase in luminosities varies from a factor of 1.3 to 3.3 and is favourable to our signal. The reduction in the effective center-of-mass energy does not have such an adverse effect on our signal as one might at first suppose; although the uncut signal cross section decreases with $\sqrt{\hat{s}}$, this is compensated by the effect of the cuts, at least at the higher energies (see Table 1). Furthermore, although bremsstrahlung is inevitable, standard, and independent of the collider designs, the beamstrahlung can always be minimized by designs, e.g. by using a ribbon-shaped beam. Thus initial state radiation has only a marginal effect in our analysis, and can even increase the signal. 
Finally, we remark briefly on the possibility of using the hadronic decays of $W Z \rightarrow$ $(j j)(j j)$, where $j$ denotes a hadronic jet, to identify the charged Higgs boson. The advantages of the hadronic mode are the much larger branching fraction and the full reconstruction of the charged Higgs boson. The increase in branching ratio is more than a factor of 25. However, the same is true for the backgrounds, and might be even worse due to additional QCD backgrounds; also it is impractical to distinguish event-by-event between the $W$ and $Z$ bosons using the hadronic mode, since they give very similar dijet invariant masses. Therefore, we have to face much larger backgrounds from $e^{+} e^{-} \rightarrow e^{+} e^{-} W^{+} W^{-}$ and $e^{+} e^{-} Z Z$. There are also complications due to the other charged Higgs bosons $H^{\prime \pm}$, which do not decay into $W Z$ but mainly into quark jets. However, if we can reconstruct the hadronic $W$ and $Z$ bosons fairly cleanly, it should still be possible to distinguish between $H^{\prime \pm}$ and $H^{ \pm}$production. This possibility might be worth exploring in the future.

In conclusion, we have investigated the feasibility of using the $W Z$ fusion process, $e^{+} e^{-} \rightarrow W^{*} Z e \nu \rightarrow H^{ \pm} e^{\mp} \nu$, to detect an exotic charged Higgs boson. If the coupling $W^{ \pm} Z H^{\mp}$ is large enough, e.g., the case when $H^{ \pm}$belongs to a Higgs-triplet and the mixing angle $s_{H}$ is close to 1 , the production of $H^{ \pm}$by $W Z$ fusion, followed by $H^{ \pm} \rightarrow W^{ \pm} Z \rightarrow$ $\ell^{ \pm} \nu \ell^{\prime} \bar{\ell}^{\prime}$, give a sizeable number of signal events above a few SM background events. In addition, we have shown that the invariant mass distribution $M(\ell \ell \ell-\min )$ is a good indicator to test for the existence of such a singly-charged triplet-Higgs boson.

Acknowledgements. Helpful discussions with Tao Han and David Miller are gratefully acknowledged. K.C. was supported in part by a DOE grant number DOE-ER-40757. 


\section{References}

[1] For proposals of a $0.5 \mathrm{TeV}-e^{+} e^{-}$collider, see e.g. " $e^{+} e^{-}$Collisions at $500 \mathrm{GeV}$ : The physics potential", DESY 92-132A.

[2] S. Komamiya, Phys. Rev. D38, 2158 (1988).

[3] J.F. Gunion, G.L. Kane, and J. Wudka, Nucl. Phys. B299, 231 (1988).

[4] M.-C Peyranère, H.E. Haber, and P. Irulegui, Phys. Rev. D44, 191 (1991).

[5] V. Barger, J. Hewett, and R.J.N. Phillips, Phys. Rev. D41, 3421 (1990); D.P. Roy, Phys. Lett. B277, 183 (1992); Phys. Lett. B283, 403 (1992); V. Barger et al., Phys. Rev. D46, 4914 (1992); R.M. Barnett et al., Phys. Rev. D47, 1048 (1993).

[6] R. Vega and D.A. Dicus, Nucl. Phys. B329, 533 (1990).

[7] V. Barger, J. Beacom, K. Cheung, and T. Han, Madison preprint (1994), MAD/PH/779, hep-ph/9404335, Phys. Rev. D50 (to appear).

[8] M.E. Peskin and T. Takeuchi, Phys. Rev. Lett. 65, 964 (1990). G. Altarelli and R. Barbieri, Phys. Lett. B253, 161 (1991).

[9] C.P. Burgess et al., Phys. Lett. B326, 276 (1994).

[10] M. Veltman, Nucl. Phys. B123, 89 (1977).

[11] P. Galison, Nucl. Phys. B232, 26 (1984).

[12] H. Georgi and M. Machacek, Nucl. Phys. B262, 463 (1985); R.S. Chivikula and H. Georgi, Phys. Lett. B182, 181 (1986).

[13] M.S. Chanowitz and M. Golden, Phys. Lett. B165, 105 (1985).

[14] J.F. Gunion, H.E. Haber, G. Kane, and S. Dawson, The Higgs Hunter's guide (Addison-Wesley, Reading, MA, 1990).

[15] J.F. Gunion, R. Vega, and J. Wudka, Phys. Rev. D42, 1673 (1990); Phys. Rev. D43, 2322 (1991). 
[16] E. Byckling and K. Kajantie, Particle Kinematics, (London, Willey, 1973).

[17] V. Barger, K. Cheung, B.A. Kniehl, and R.J.N. Phillips, Phys. Rev. D46, 3725 (1992).

[18] K. Hagiwara, J. Kanzaki, and H. Muryama, Durham preprint (1991), DTP-91-18.

[19] V. Barger, J. Ohnemus, and R.J.N. Phillips, Phys. Rev. D35, 166 (1987).

[20] P. Chen, T.L. Barklow, and M.E. Peskin, in proceedings of workshop on "Physics and Experiments with Linear $e^{+} e^{-}$Colliders", Waikoloa, Hawaii (April 1993). 


\section{Figure and Table Captions}

Fig. 1: Feynman diagrams responsible for producing the singly-charged Higgs-triplet boson: (i) $e^{+} e^{-} \rightarrow Z^{*} \rightarrow W^{-} H^{+}$and (ii) $e^{+} e^{-} \rightarrow W^{+*} Z e^{-} \bar{\nu}_{e} \rightarrow H^{+} e^{-} \bar{\nu}_{e}$.

Fig. 2: Production cross section of the charged Higgs-triplet boson via the Bjorkentype process $e^{+} e^{-} \rightarrow Z^{*} \rightarrow W^{\mp *} H^{ \pm} \rightarrow f \bar{f}^{\prime} H^{ \pm}$for different c.m.s. energies: $\sqrt{s}=500 \mathrm{GeV}$ (solid line), $1 \mathrm{TeV}$ (dashed line), 1.5 TeV (dash-dotted line), and $2 \mathrm{TeV}$ (dotted line). It is summed over all possible $f \bar{f}^{\prime}$ pairs.

Fig. 3: Production cross section of the charged Higgs-triplet boson via the $W Z$ fusion process $e^{+} e^{-} \rightarrow H^{ \pm} e^{\mp} \nu$ for c.m.s. energies $\sqrt{s}=500 \mathrm{GeV}$ (solid line), $1 \mathrm{TeV}$ (dashed line), 1.5 TeV (dash-dotted line) and $2 \mathrm{TeV}$ (dotted line).

Fig. 4: Histogram estimates indicating the excess of the leptonic signal from $W Z \rightarrow$ $H^{ \pm}$fusion (shaded area) above the background at $\sqrt{s}=1.5 \mathrm{TeV}$, as a function of the leptonic invariant mass $M(l l l-\min )$ defined in the text, with charged Higgs-boson masses: (a) $M_{H}=200 \mathrm{GeV}$, (b) $M_{H}=300 \mathrm{GeV}$, (c) $M_{H}=$ $400 \mathrm{GeV}$, and (d) $M_{H}=500 \mathrm{GeV}$.

Tab. 1: Production cross section $\sigma / s_{H}^{2}$ (in $\mathrm{fb}$ ) of charged Higgs bosons in the channel $e^{+} e^{-} \rightarrow H^{ \pm *} e^{\mp} \nu \rightarrow W^{ \pm} Z e^{\mp} \nu ; W \rightarrow e \nu, \mu \nu, e \nu \nu \nu, \mu \nu \nu \nu$, and $Z \rightarrow e e, \mu \mu$ as a function of $M_{H}$ after the kinematic cuts discussed in the text. At the end of the table, we also present results for the background processes (a), (b), and $(\mathrm{c})$ in $\mathrm{fb}$. 
Table 1

\begin{tabular}{|c|c|c|c|c|}
\hline$M_{H}[\mathrm{GeV}]$ & $\sqrt{s}=0.5 \mathrm{TeV}$ & $\sqrt{s}=1 \mathrm{TeV}$ & $\sqrt{s}=1.5 \mathrm{TeV}$ & $\sqrt{s}=2 \mathrm{TeV}$ \\
\hline Signal & & & & \\
\hline 175 & 0.19 & 0.43 & 0.41 & 0.32 \\
\hline 200 & 0.15 & 0.40 & 0.39 & 0.31 \\
\hline 250 & 0.10 & 0.35 & 0.36 & 0.29 \\
\hline 300 & 0.05 & 0.29 & 0.34 & 0.28 \\
\hline 350 & 0.02 & 0.24 & 0.31 & 0.28 \\
\hline 400 & & 0.21 & 0.27 & 0.25 \\
\hline 500 & & 0.12 & 0.22 & 0.22 \\
\hline 600 & & 0.06 & 0.17 & 0.18 \\
\hline 700 & & 0.02 & 0.12 & 0.15 \\
\hline 800 & & & 0.08 & 0.12 \\
\hline Background & & & & \\
\hline (a) & 0.04 & 0.08 & 0.07 & 0.06 \\
\hline (b) & 0.03 & 0.05 & 0.05 & 0.05 \\
\hline (c) & 0.01 & 0.08 & 0.17 & 0.23 \\
\hline Total & 0.08 & 0.21 & 0.29 & 0.34 \\
\hline
\end{tabular}




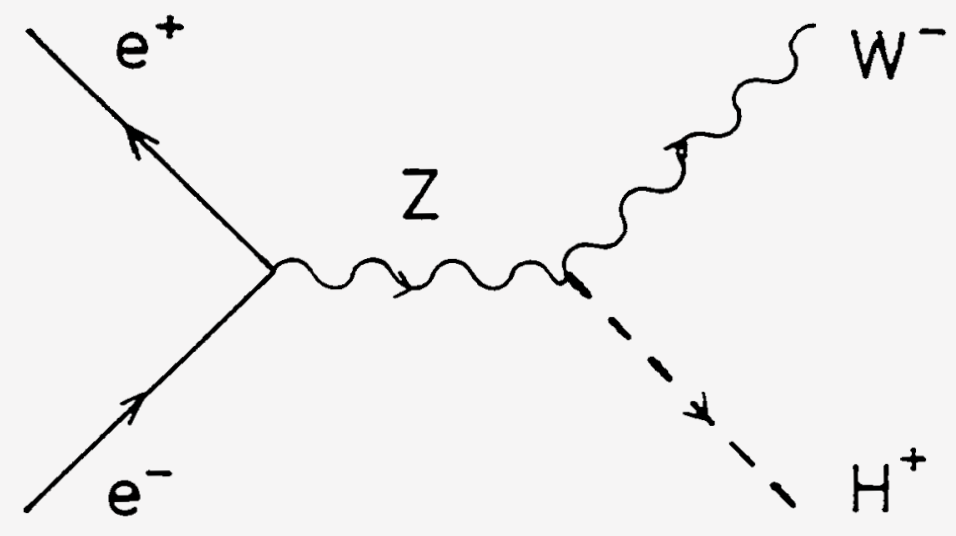

(i)

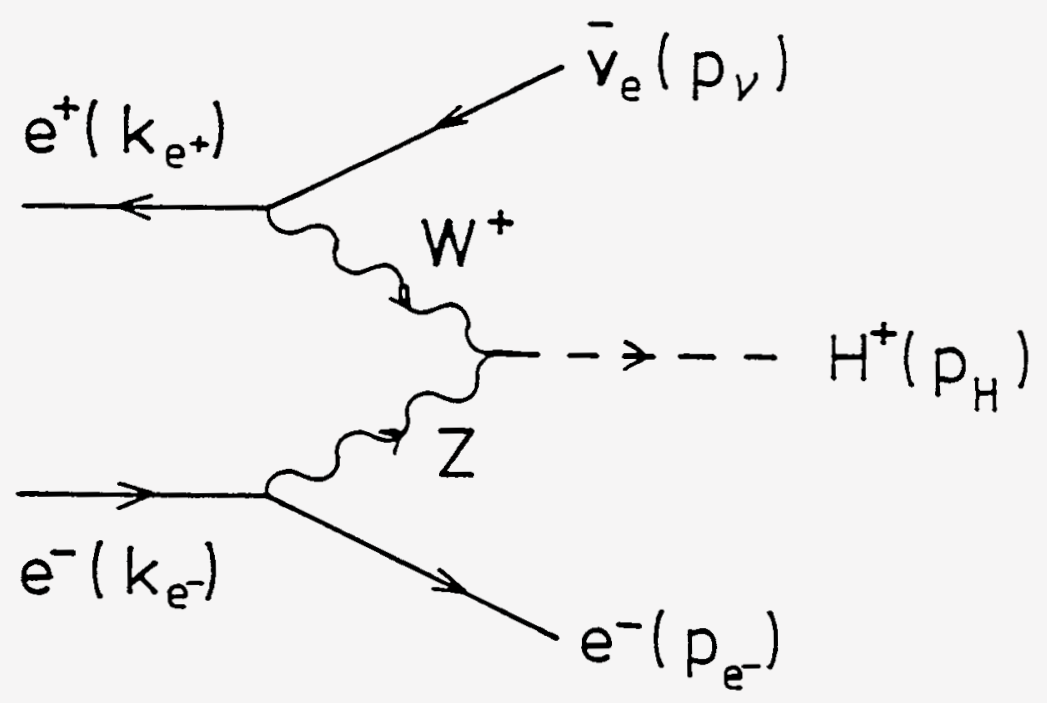

(ii)

Fig. 1 


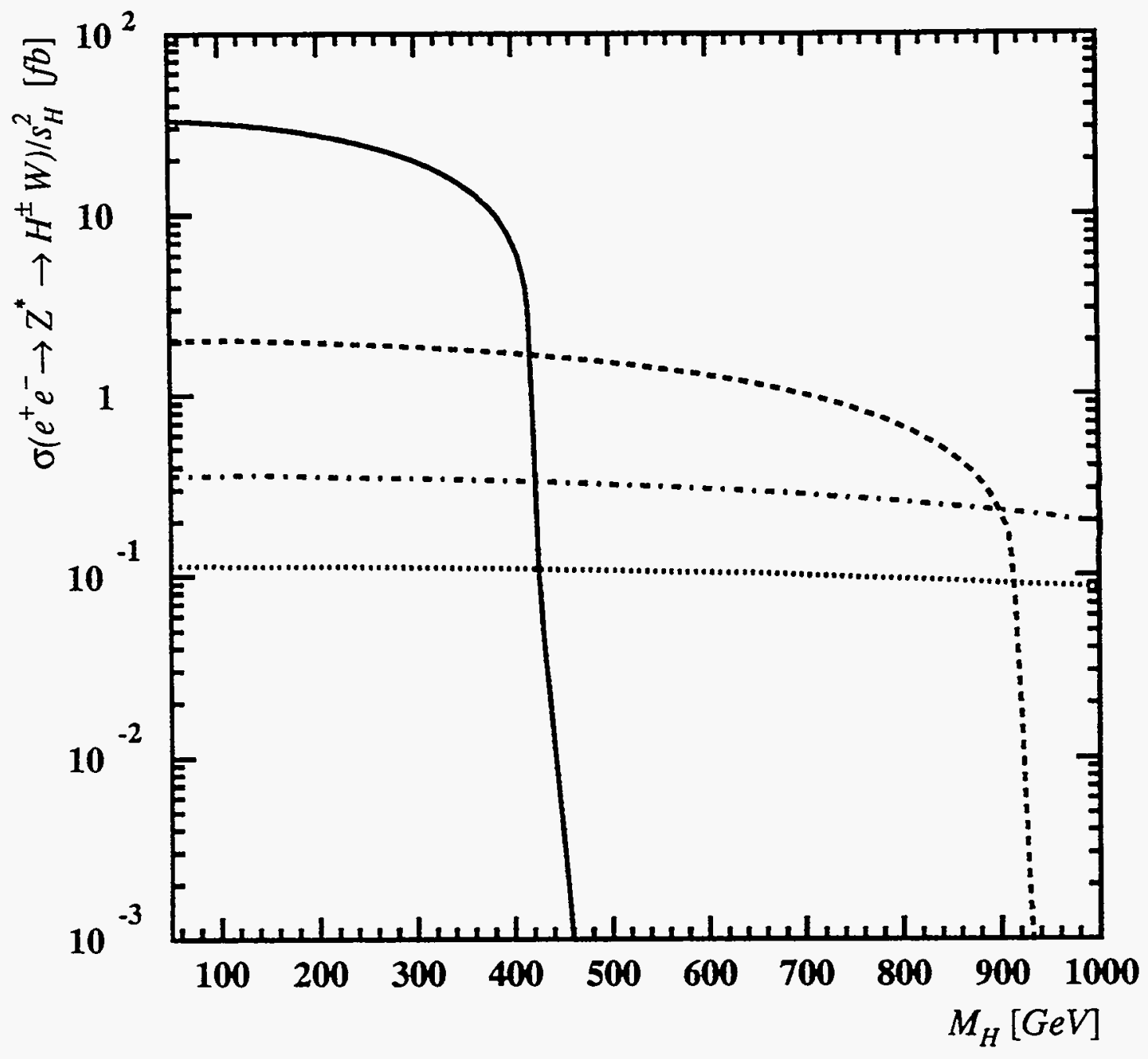

Fig. 2 


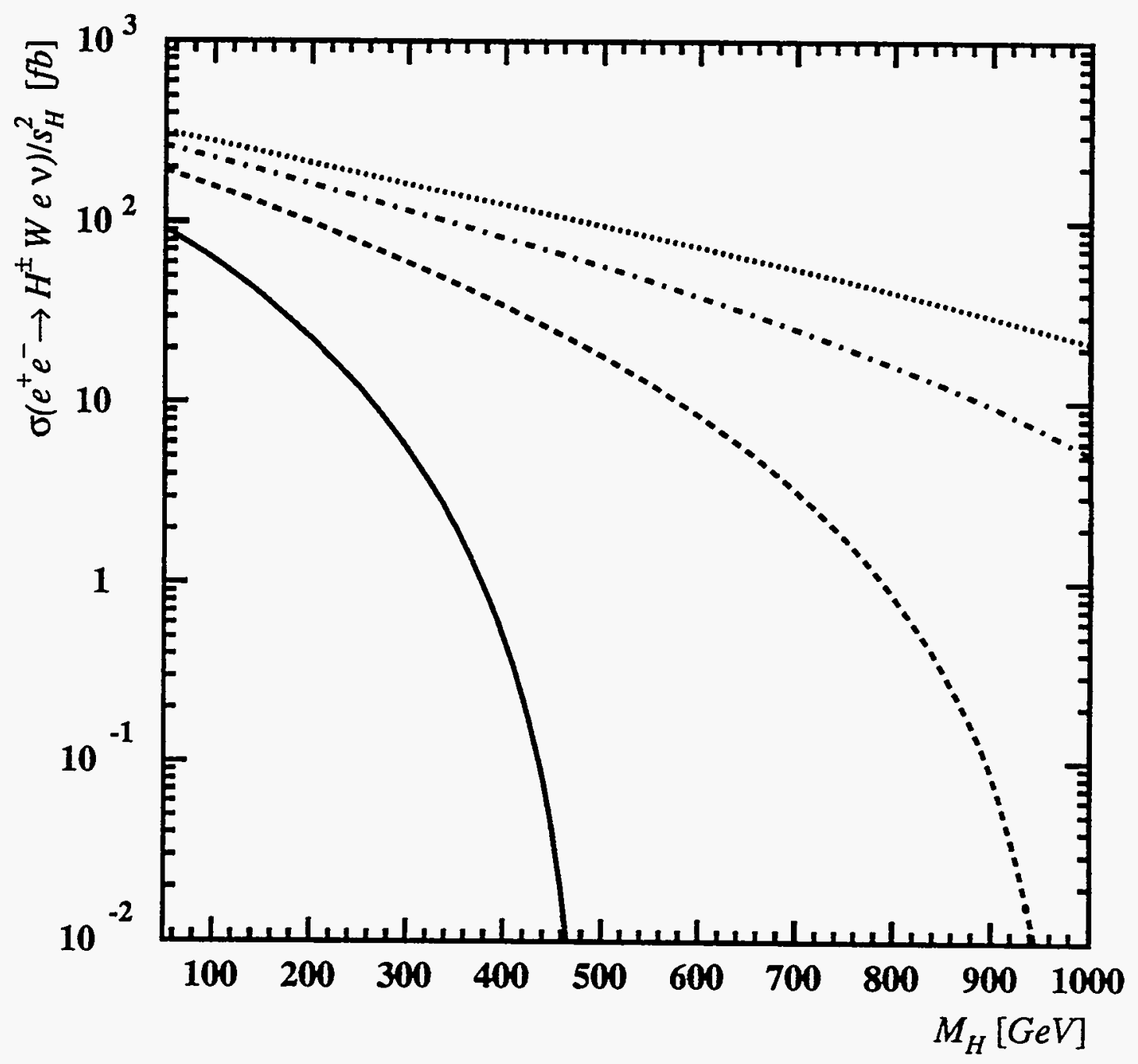

Fig. 3 

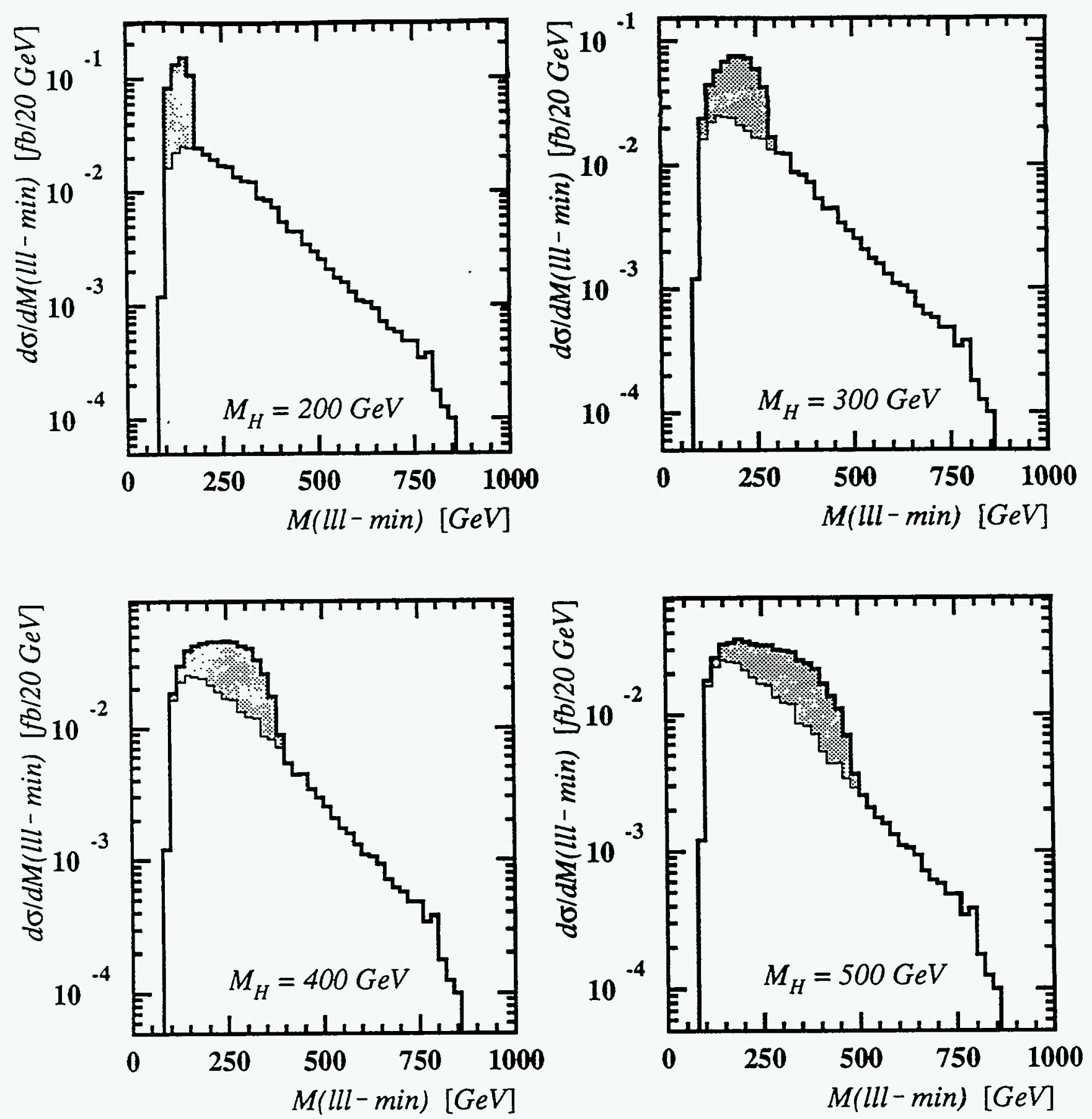

Fig. 4 